\title{
Extramedullary plasmacytoma of maxilla with cervical nodal metastasis associated with sinonasal aspergillosis
}

\author{
Virender Suhag1, Sunita BS², Ajaya Kumar Singh ${ }^{3}$, Sunita Dashottar ${ }^{4}$ \\ ${ }^{I}$ Department of Radiation Oncology, Classified Specialist Pathology, Command Hospital (CC), Lucknow, India \\ ${ }^{2}$ Department of Pathology, Classified Specialist Pathology, Command Hospital (CC), Lucknow, India \\ ${ }^{3}$ Department of Radiodiagnosis, Army Hospital $(R \& R)$, Delhi, India \\ ${ }^{4}$ Department of Radiodiagnosis, Military Hospital (CTC), Pune, India
}

Received March 13, 2015; Revised September 23, 2015; Accepted September 26, 2015; Published Online September 28,2015

\section{Case Report}

\begin{abstract}
Extramedullary plasmacytoma (EMP) is defined as neoplastic proliferation of plasma cells in the soft tissue. It represents approximately $3 \%$ of all plasma cell neoplasms. EMP of the head and neck are very uncommon tumors, representing approximately $0.4 \%$ of all head and neck malignancies; amongst them, plasmacytoma of the maxilla is extremely rare. We present a rare case of EMP which had metastasized to multiple cervical lymph nodes and was associated with aspergillosis. The possibility of extramedullary plasmacytoma should be considered when an elderly patient presents with symptoms of recurrent rhinosinusitis not responding to conservative management and imaging shows a destructive soft tissue mass.
\end{abstract}

Keywords: Extra Medullary Plasmacytoma; Maxilla; Aspergillosis

\section{Introduction}

Plasma cell neoplasms are a group of clinical disorders characterized by an uncontrolled monoclonal proliferation of plasma cells. Localized forms of plasma cells neoplasms are solitary bone plasmacytoma (SBP) and extramedullary plasmacytoma (EMP); multiple myeloma (MM) is the disseminated type of this disorder. ${ }^{1}$ EMP is defined as neoplastic proliferation of plasma cells in the soft tissue. It represents approximately $3 \%$ of all plasma cell neoplasms. EMP of the head and neck is very uncommon tumor, representing approximately $0.4 \%$ of all head and neck malignancies; amongst them, plasmacytoma of the maxilla is extremely rare.,3 EMP occurs approximately three times more often in men than in women, usually seen in age group 50-70 years. Regional lymph nodes are invaded in less than $10 \%$ of EMP and $16 \%$ of patients progress to multiple myeloma. ${ }^{1,3,4}$

Our case of EMP is extremely rare in the sense that it had metastasized to multiple cervical lymph nodes and was associated with aspergillosis. Megat Shiraz $\mathrm{MA}^{1}$ reported a case of EMP of the right maxillary sinus, which had metastasized to the brain and rib. Shyan Gau JS ${ }^{2}$ described a 68-year-old patient who developed an EMP coexisting with fungus balls in the paranasal sinuses (PNS) and was initially treated as fungal sinusitis.

\section{Case presentation}

A 68 years old male presented with the chief complaints of pain right side of jaw and right temporal region associated with nasal stuffiness of about two years duration. His initial computed tomography (CT) scan of face and neck done two years ago was suggestive of expansile homogeneous soft tissue density lesion in right maxilla with bone erosions; and biopsy of maxillary mass lesion had revealed chronic non specific inflammation. He was managed conservatively in otorhinolaryngology outpatient department as a case of chronic rhinosinusitis associated with nasal polyp in right mid meatus. He received a short course of oral systemic steroid in the form of $50 \mathrm{mg}$ of prednisolone daily followed by topical budesonide and oral flucortolone; along with broad spectrum antibiotic amoxy/clavulinic acid and supportive care by topical decongestant oxymetazoline, nasal saline irrigation and steam inhalation.

His symptoms persisted and his repeat CT scan of PNS (Figure 1) was suggestive of soft tissue density material filling right maxillary sinus and right ethmoidal air cells; ostium blocked with inspissated material. He underwent functional endoscopic sinus surgery (FESS) evacuation. The lesion was excised; gross examination revealed a polypoidal mass with fungal ball while the histopathology showed septate hyphae branching at acute angle, which is characteristic of 
aspergillosis, admixed with chronic inflammation. $\mathrm{He}$ received antifungal treatment in the form of intravenous Amphotericin B followed by oral Voreconazole for a total of 2 weeks. Two months post excision; the patient had recurrence of symptoms and developed multiple cervical lymph node enlargements. Fine needle aspiration cytology of cervical lymph nodes was suggestive of metastatic deposits from Plasmacytoma. Repeat CT scan of PNS showed mildly enhancing soft tissue mass in right maxillary sinus with erosion of all its walls and extension into right nasal cavity, right infra-temporal fossa, right ethmoid \& sphenoid sinuses, and right orbit. In view of cytological evidence of malignancy, the mass was upfront excised without subjecting the patient to further supportive and conservative management. Excision of mass was done. Histopathological examination of the excised mass (Figure 2) confirmed extramedullary plasmacytoma with chronic granulomatous reaction. Immunohistochemistry was positive for kappa and negative for CK, LCA, CD20 and lambda in the malignant cells.

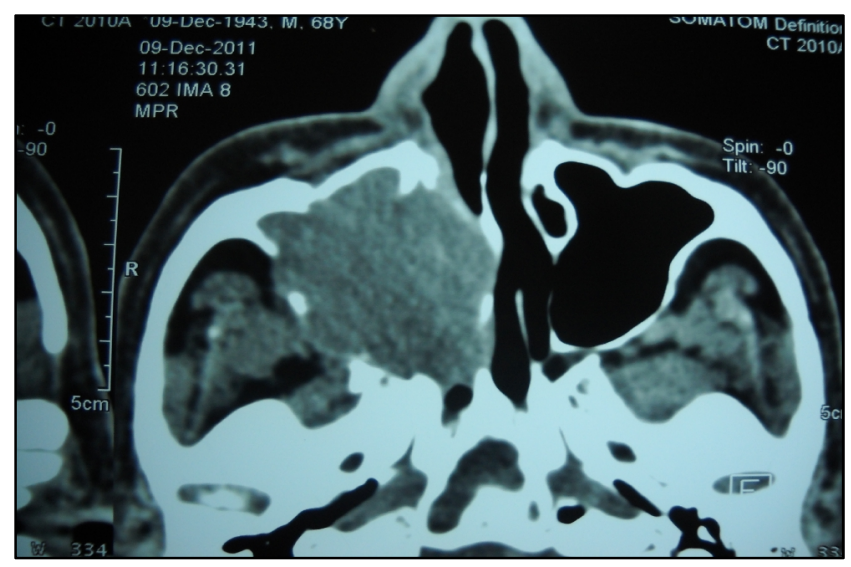

FIG. 1: CT PNS showing right maxillary mass causing bone erosion.

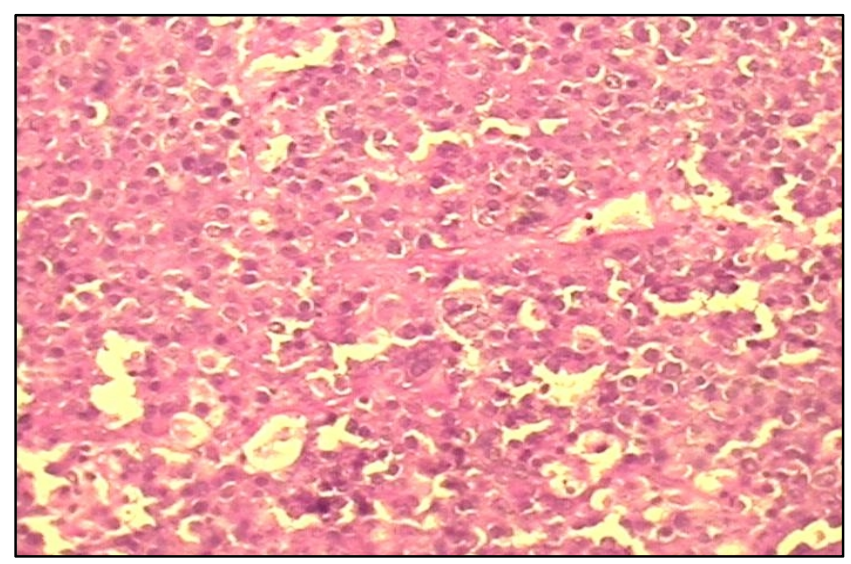

FIG. 2: Excised maxillary mass showing sheets of plasma cells.

The patient underwent thorough evaluation to exclude multiple myeloma, including skeletal survey, Bence Jones protein assessment, protein electrophoresis, bone marrow aspiration and biopsy. All these investigations were within normal limits. Based on these clinical, radiological and biochemical parameters, a final diagnosis of Extramedullary plasmacytoma of right maxilla associated with cervical lymph nodal metastasis and chronic fungal sinusitis was made. The patient received external beam radiotherapy for a dose of $46 \mathrm{~Gy}$ in 23 fractions by involved field radiotherapy technique on a Telecobalt machine; and chemotherapy in view of metastatic cervical nodes and showed good response. He has shown 1 year disease free interval when last seen.

\section{Discussions}

Extramedullary plasmacytoma arising from the maxillary sinus with metastasis is extremely rare. ${ }^{1,5,6}$ Local clinical signs and symptoms are bone swelling, localized pain in the jaws and teeth, numbness, anesthesia, bleeding, tooth mobility and possible bone fracture. Very rarely, it may mimic an abscess. ${ }^{7}$ Radiographically EMP is seen as a well defined uni or multilocular lytic lesion without periosteal reaction, or as a protruding mass with cortical expansion. The diagnosis of plasmacytoma is based on and confirmed with histology and immune-histochemistry. The current criteria to diagnose EMP include isolated area of bone destruction due to clonal plasma cells, bone marrow plasma cell infiltration not exceeding $5 \%$ of all nucleated cells, and absence of further osteolytic bone lesions or other tissue involvement (i.e., no evidence of systemic plasmacytoma). Systemic findings related to the production of monoclonal protein are usually not present and a monoclonal spike in serum electrophoresis may be absent as is the monoclonal Bence-Jones protein in the urine. Other systemic alterations as hypocalcaemia, hypokalemia, anemia, leucopenia, thrombocytopenia, and impaired renal function are absent in EMP, but are usually present in multiple myeloma. ${ }^{1,3}$

Localized extramedullary plasmacytoma is highly radiosensitive and the recommended primary treatment for localized extramedullary plasmacytoma is radical radiotherapy up to doses of $40-50 \mathrm{~Gy} .{ }^{3}$ Surgical treatment is elective in those selected cases where all tumor is removed with minimal cosmetic or functional deficit or those to prevent or stabilize a pathologic mandibular fracture. For generalized extramedullary plasmacytoma, chemotherapy is advisable. Even though extramedullary plasmacytoma of the maxillary sinus is rare, it should be one of the differential diagnoses for maxillary tumors. Metastasis from other primary sites must be ruled out clinically and radiologically as unusual bone metastasis may rarely present as primary bone malignancy. ${ }^{8}$

The cooperation among the otorhinolaryngologists, pathologists and hematologists is required to optimally manage extramedullary plasmacytoma patients. ${ }^{1}$ Patients should be followed by periodically to rule out progression to multiple myeloma. The blood tests include hemogram, blood urea nitrogen, serum creatinine, serum calcium, LDH, Beta2 
microglobulin assay, serum protein electrophoresis, serum immunofixation electrophoresis and serum free light chain assay. Urine tests include 24 hour urine assay for total proteins, urine plasma electrophoresis and urine immunofixation electrophoresis. PET-CT scan, skeletal survey and bone marrow biopsy should be considered at least annually or as clinically indicated. ${ }^{9-11}$ The prognosis is more favorable in patients with primary EMP not associated with MM. Although a small proportion of these patients have a rapidly progressive course with dissemination and death in a few months, about 33-75\% survive for five years. ${ }^{4}$ To conclude, the possibility of extramedullary plasmacytoma should be considered when an elderly patient presents with symptoms of recurrent rhinosinusitis not responding to conservative management and CT shows a destructive soft tissue mass.

\section{Conflict of interest}

The authors declare that they have no conflicts of interest. The authors alone are responsible for the content and writing of the paper.

\section{References}

1. Megat Shiraz MA, Jong YH, Primuharsa Putra SH. Extramedullary plasmacytoma in the maxillary sinus. Singapore Med J. 2008;49:e310-1.

2. Gau JS, Lu NH, Yeh LR, et al. Coexistence of extramedullary plasmacytoma and fungus balls in the paranasal sinuses. J Med Cases. 2011; 2: 162-5.

3. Karmponis P, Kamargiannis N, Tigiroglou E, et al. Maxillary sinus plasmacytoma - a case report. Head Neck Surg. 2011; 46: 45-8.

4. Chowdhary A, Bhagat DR, Hamid S, et al. Extramedullary plasmacytoma of maxillary antrum. JK Science. 2009; 11: 44-6.

5. Korolkowa O, Osuch-Wójcikiewicz E, Deptała A, Suleiman W. Extramedullary plasmacytoma of the head and neck. Otolaryngol Pol. 2004;58:1009-12.

6. Kamal M, Kaur P, Gupta R, Gupta S, Singh S. Mandibular plasmacytoma of jaw - a case report. J Clin Diagn Res. 2014;8:ZD20-1.

7. Nanda KD, Bhargava D, Sharma B, Dave A. Plasmacytoma masquerading as an abscess. J Investig Clin Dent. 2012;3:236-9.
8. Varshney S, Pant HC. Unusual bone metastasis from follicular carcinoma thyroid: presenting as a primary bone malignancy. Int J Cancer Ther Oncol. 2014; 2:020411.

9. Tikku G, Jain M, Mridha A, Grover R. Solitary bone plasmacytoma progressing into retroperitoneal plasma cell myeloma with no related end organ or tissue impairment: A case report and review of the literature. Turk J Haematol. 2014;31:286-9.

11. Jawad MU, Scully SP. Skeletal Plasmacytoma: progression of disease and impact of local treatment; an analysis of SEER database. J Hematol Oncol. 2009; 2:41.

12. Kilciksiz S, Karakoyun-Celik O, Agaoglu FY, Haydaroglu A. A review for solitary plasmacytoma of bone and extramedullary plasmacytoma. ScientificWorldJournal. 2012;2012:895765. 